\title{
Physics of Stars and the Measurement Data: Part III
}

\author{
Boris V.Vasiliev
}

Independent Researcher, Russia

*Corresponding Author: bv.vasiliev@ narod.ru

Copyright (c)2014 Horizon Research Publishing All rights reserved.

\begin{abstract}
The explanation of dependencies of the parameters of the stars and the Sun which was measured by astronomers is considered as a main task of the physics of stars. This theory is based on taking into account of the existence of a gravity-induced electric polarization of intrastellar plasma because this plasma is an electrically polarized substance. The accounting of the gravity-induced electric polarization gives the explanation to data of astronomical measurements: the temperature-radius-mass-luminosity relations, the spectra of seismic oscillations of the Sun, distribution of stars on their masses, magnetic fields of stars and etc. The stellar radiuses, masses and temperatures are expressed by the corresponding ratios of the fundamental constants, and individuality of stars are determined by two parameters - by the charge and mass numbers of nuclei, from which a stellar plasma is composed. This theory is the lack of a collapse in the final stage of the star development, as well as "black holes" that could be results from a such collapse.
\end{abstract}

Keywords Electric Polarization, Plasma, Stellar Mass, Stellar Temperature, Stellar Radius, Seismic Oscillations, Magnetic Field

PACS: $64.30 .+\mathrm{i} ;$ 95.30.-k

\section{A mechanism of stabilization for neutron-excess nuclei in plasma}

\subsection{Neutron-excess nuclei and the neutroniza- tion}

The form of the star mass spectrum (Fig.1, part I) indicates that plasma of many stars consists of neutron-excess nuclei with the ratio $A / Z=3,4,5$ and so on. These nuclei are subjects of a decay under the "terrestrial" conditions. Hydrogen isotopes ${ }_{1}^{4} \mathrm{H},{ }_{1}^{5} \mathrm{H},{ }_{1}^{6} \mathrm{H}$ have short time of life and emit electrons with energy more than $20 \mathrm{Mev}$. The decay of helium isotopes ${ }_{2}^{6} \mathrm{He},{ }_{2}^{8} \mathrm{He},{ }_{2}^{10} \mathrm{He}$ have the times of life, which can reach tenth part of the seconds.

Stars have the time of life about billions years and the lines of their spectrum of masses are not smoothed. Thus we should suppose that there is some mechanism of stabilization of neutron-excess nuclei inside stars. This mechanism is well known - it is neutronization [1], $§ 106$. It is accepted to think that this mechanism is characteristic for dwarfs with density of particles about $10^{30}$ per $\mathrm{cm}^{3}$ and pressure of relativistic electron gas

$$
P \approx \hbar c \cdot n_{e}^{4 / 3} \approx 10^{23} \text { dyne } / \mathrm{cm}^{2} .
$$

The possibility of realization of neutronization in dense plasma is considered below in detail. At thus, we must try to find an explanation to characteristic features of the star mass spectrum. At first, we can see that, there is actually quite a small number of stars with $A / Z=2$ exactly. The question is arising: why there are so few stars, which are composed by very stable nuclei of helium- 4 ? At the same time, there are many stars with $A / Z=4$, i.e. consisting apparently of a hydrogen-4, as well as stars with $A / Z=3 / 2$, which hypothetically could be composed by another isotope of helium helium-3.

\subsubsection{The electron cloud of plasma cell}

Let us consider a possible mechanism of the action of the electron gas effect on the plasma nuclear subsystem. It is accepted to consider dense plasma to be divided in plasma cells. These cells are filled by electron gas and they have positively charged nuclei in their centers [2].

This construction is non stable from the point of view of the classical mechanics because the opposite charges collapse is "thermodynamic favorable". To avoid a divergence in the theoretical description of this problem, one can artificially cut off the integrating at some small distance characterizing the particles interaction. For example, nuclei can be considered as hard cores with the finite radii.

It is more correctly, to consider this structure as a quantummechanical object and to suppose that the electron can not approach the nucleus closer than its own de Broglie's radius $\lambda_{e}$.

Let us consider the behavior of the electron gas inside the plasma cell. If to express the number of electrons in the volume $V$ through the density of electron $n_{e}$, then the maximum value of electron momentum [1]:

$$
p_{F}=\left(3 \pi^{2} n_{e}\right)^{1 / 3} \hbar \text {. }
$$

The kinetic energy of the electron gas can be founded from the general expression for the energy of the Fermi-particles, which fills the volume $V$ [1]:

$$
\mathcal{E}=\frac{V c}{\pi^{2} \hbar^{3}} \int_{0}^{p_{F}} p^{2} \sqrt{m_{e}^{2} c^{2}+p^{2}} d p
$$


After the integrating of this expression and the subtracting of the energy at rest, we can calculate the kinetic energy of the electron:

$$
\begin{array}{r}
\mathcal{E}_{\text {kin }}=\frac{3}{8} m_{e} c^{2} . \\
\cdot\left[\frac{\xi\left(2 \xi^{2}+1\right) \sqrt{\xi^{2}+1}-\operatorname{Arcsinh}(\xi)-\frac{8}{3} \xi^{3}}{\xi^{3}}\right]
\end{array}
$$

(where $\xi=\frac{p_{F}}{m_{e} c}$ ).

The potential energy of an electron is determined by the value of the attached electric field. The electrostatic potential of this field $\varphi(r)$ must be equal to zero at infinity. ${ }^{1}$ With this in mind, we can write the energy balance equation of electron

$$
\mathcal{E}_{\text {kin }}=e \varphi(r) .
$$

The potential energy of an electron at its moving in an electric field of the nucleus can be evaluated on the basis of the Lorentz transformation [3],§24. If in the laboratory frame of reference, where an electric charge placed, it creates an electric potential $\varphi_{0}$, the potential in the frame of reference moving with velocity $v$ is

$$
\varphi=\frac{\varphi_{0}}{\sqrt{1-\frac{v^{2}}{c^{2}}}} .
$$

Therefore, the potential energy of the electron in the field of the nucleus can be written as:

$$
\mathcal{E}_{\text {pot }}=-\frac{Z e^{2}}{r} \frac{\xi}{\beta}
$$

Where

$$
\beta=\frac{v}{c}
$$

and

$$
\xi \equiv \frac{p}{m_{e} c},
$$

$m_{e}$ is the mass of electron in the rest.

And one can rewrite the energy balance Eq.(5) as follows:

$$
\frac{3}{8} m_{e} c^{2} \xi \mathbb{Y}=e \varphi(r) \frac{\xi}{\beta}
$$

where

$$
\mathbb{Y}=\left[\frac{\xi\left(2 \xi^{2}+1\right) \sqrt{\xi^{2}+1}-\operatorname{Arcsinh}(\xi)-\frac{8}{3} \xi^{3}}{\xi^{4}}\right]
$$

Hence

$$
\varphi(r)=\frac{3}{8} \frac{m_{e} c^{2}}{e} \beta \mathbb{Y}
$$

In according with Poisson's electrostatic equation

$$
\Delta \varphi(r)=4 \pi e n_{e}
$$

or at taking into account that the electron density is depending on momentum (Eq.(2)), we obtain

$$
\Delta \varphi(r)=\frac{4 e}{3 \pi}\left(\frac{\xi}{\bar{\lambda}_{C}}\right)^{3},
$$

\footnotetext{
${ }^{1}$ In general, if there is an uncompensated electric charge inside the cell, then we would have to include it to the potential $\varphi(r)$. However, we can do not it, because will consider only electro-neutral cell, in which the charge of the nucleus exactly offset by the electronic charge, so the electric field on the cell border is equal to zero.
}

where $\chi_{C}=\frac{\hbar}{m_{e} c}$ is the Compton radius.

At introducing of the new variable

$$
\varphi(r)=\frac{\chi(r)}{r}
$$

we can transform the Laplacian:

$$
\Delta \varphi(r)=\frac{1}{r} \frac{d^{2} \chi(r)}{d r^{2}} .
$$

As (Eq.(12))

$$
\chi(r)=\frac{3}{8} \frac{m_{e} c^{2}}{e} \mathbb{Y} \beta r,
$$

the differential equation can be rewritten:

$$
\frac{d^{2} \chi(r)}{d r^{2}}=\frac{\chi(r)}{\mathbb{L}^{2}}
$$

where

$$
\mathbb{L}=\left(\frac{9 \pi}{32} \frac{\mathbb{Y} \beta}{\alpha \xi^{3}}\right)^{1 / 2} \chi_{C}
$$

$\alpha=\frac{1}{137}$ is the fine structure constant.

With taking in to account the boundary condition, this differential equation has the solution:

$$
\chi(r)=C \cdot \exp \left(-\frac{r}{\mathbb{L}}\right) .
$$

Thus, the equation of equilibrium of the electron gas inside a cell (Eq.(10)) obtains the form:

$$
\frac{Z e}{r} \cdot e^{-r / \mathbb{L}}=\frac{3}{8} m_{e} c^{2} \beta \mathbb{Y} .
$$

\subsection{The Thomas-Fermi screening}

Let us consider the case when an ion is placed at the center of a cell, the external shells don't permit the plasma electron to approach to the nucleus on the distances much smaller than the Bohr radius. The electron moving is non-relativistic in this case. At that $\xi \rightarrow 0$, the kinetic energy of the electron

$$
\mathcal{E}_{\text {kin }}=\frac{3}{8} m_{e} c^{2} \xi \mathbb{Y} \rightarrow \frac{3}{5} E_{F}
$$

and the screening length

$$
\mathbb{L} \rightarrow \sqrt{\frac{\mathcal{E}_{F}}{6 \pi e^{2} n_{e}}} .
$$

Thus, we get the Thomas-Fermi screening in the case of the non-relativistic motion of an electron.

\subsection{The screening with relativistic electrons}

In the case the $\ll$ bare $\gg$ nucleus, there is nothing to prevent the electron to approach it at an extremely small distance $\lambda_{\text {min }}$, which is limited by its own than its de Broglie's wavelength. Its movement in this case becomes relativistic at $\beta \rightarrow 1 \xi \gg 1$. In this case, at not too small $\xi$, we obtain

$$
\mathbb{Y} \approx 2\left(1-\frac{4}{3 \xi}\right)
$$


and at $\xi \gg 1$

$$
\mathbb{Y} \rightarrow 2
$$

In connection with it, at the distance $r \rightarrow \lambda_{\text {min }}$ from a nucleus, the equilibrium equation (21) reforms to

$$
\lambda_{\text {min }} \simeq Z \alpha \lambda_{C}
$$

and the density of electron gas in a layer of thickness $\lambda_{\min }$ can be determined from the condition of normalization. As there are $\mathrm{Z}$ electrons into each cell, so

$$
Z \simeq n_{e}^{\lambda} \cdot \lambda_{\min }^{3}
$$

From this condition it follows that

$$
\xi_{\lambda} \simeq \frac{1}{2 \alpha Z^{2 / 3}}
$$

Where $n_{e}^{\lambda}$ and $\xi_{\lambda}$ are the density of electron gas and the relative momentum of electrons at the distance $\lambda_{\min }$ from the nucleus. In accordance with Eq.(5), the energy of all the $\mathrm{Z}$ electrons in the plasma cell is

$$
\mathcal{E} \simeq Z m_{e} c^{2} \xi_{\lambda}
$$

At substituting of Eq.(28), finally we obtain the energy of the electron gas in a plasma cell:

$$
\mathcal{E} \simeq \frac{m_{e} c^{2}}{2 \alpha} Z^{1 / 3}
$$

This layer provides the pressure on the nucleus:

$$
P \simeq \mathcal{E}\left({\frac{\xi}{\lambda_{C}}}^{3} \approx 10^{23} \text { dyne } / \mathrm{cm}^{2}\right.
$$

This pressure is in order of value with pressure of neutronization (1).

Thus the electron cloud forms a barrier at a distance of alpha left $\left(\frac{h b a r}{m_{e} c}\right.$ right $)$ from the nucleus. This barrier is characterized by the energy:

$$
\mathcal{E} \simeq c p^{\max } \simeq \frac{m_{e} c^{2}}{\alpha} \approx 70 M e v .
$$

This energy is many orders of magnitude more energy characteristic of the electron cloud on the periphery of the plasma cells, which we have neglected for good reason.

The barrier of the electron cloud near the nucleus will prevent its $\beta$-decay, if it has less energy. As a result, the nucleus that exhibit $\beta$-activity in the atomic matter will not disintegrate in plasma.

Of course, this barrier can be overcome due to the tunnel effect. The nuclei with large energy of emitted electrons have more possibility for decay. Probably it can explain the fact that the number of stars, starting with the $A / Z \approx 4$ spectrum (Fig.(Part I-1)), decreases continuously with increasing $A / Z$ and drops to zero when $A / Z \approx 10$, where probably the decaying electron energy is approaching to the threshold Eq.(32).

It is interesting whether is possible to observe this effect in the laboratory? It seems that one could try to detect a difference in the rate of decay of tritium nuclei adsorbed in a metal. Hydrogen adsorbed in various states with different metals [4]. Hydrogen behaves like a halogen in the alkali metals ( $\mathrm{Li}, \mathrm{K})$. It forms molecules $\mathrm{H}^{-} \mathrm{Li}^{+}$and $\mathrm{H}^{-} \mathrm{K}^{+}$with an absorbtion of electron from electron gas. Therefore, the tritium decay rate in such metals should be the same as in a molecular gas. However assumed [4], that adsorbed hydrogen is ionized in some metals, such as $T i$, and it exists there in the form of gas of "naked" nuclei. In this case, the free electrons of the metal matrix should form clouds around the bare nuclei of tritium. In accordance with the above calculations, they should suppress the $\beta$-decay of tritium.

\subsection{The neutronization}

. The considered above $\ll$ attachment $\gg$ of the electron to the nucleus in a dense plasma should lead to a phenomenon neutronization of the nucleus, if it is energetically favorable. The $\ll$ attached $\gg$ electron layer should have a stabilizing effect on the neutron-excess nuclei, i.e. the neutron-excess nucleus, which is instable into substance with the atomic structure, will became stable inside the dense plasma. It explains the stable existence of stars with a large ratio of $\mathrm{A} / \mathrm{Z}$.

These formulas allow to answer questions related to the characteristics of the star mass distribution. The numerical evaluation of energy the electron gas in a plasma cell gives:

$$
\mathcal{E} \simeq \frac{m_{e} c^{2}}{2 \alpha} Z^{1 / 3} \approx 5 \cdot 10^{-5} Z^{1 / 3} \operatorname{erg}
$$

The mass of nucleus of helium- $4 \mathrm{M}\left({ }_{2}^{4} \mathrm{He}\right)=4.0026$ a.e.m., and the mass hydrogen- $4 M\left({ }_{1}^{4} H\right)=4.0278$ a.e.m.. The mass defect $\approx 3.8 \cdot 10^{-5}$ egr. Therefore, the reaction

$$
{ }_{2}^{4} H e+e \rightarrow{ }_{1}^{4} H+\widetilde{\nu},
$$

is energetically favorable. At this reaction the nucleus captures the electron from gas and proton becomes a neutron.

There is the visible line of stars with the ratio $A / Z=3 / 2$ at the star mass spectrum. It can be attributed to the stars, consisting of ${ }_{2}^{3} \mathrm{He},{ }_{4}^{6} \mathrm{Be},{ }_{6}^{9} \mathrm{C}$, etc.

It is not difficult to verify at direct calculation that the reactions of neutronization and transforming of ${ }_{2}^{3} \mathrm{He}$ into ${ }_{1}^{3} \mathrm{H}$ and ${ }_{4}^{6} \mathrm{Be}$ into ${ }_{3}^{6} \mathrm{Li}$ are energetically allowed. So the nuclei ${ }_{2}^{3} \mathrm{He}$ and ${ }_{4}^{6} \mathrm{Be}$ should be conversed by neutronization into ${ }_{1}^{3} \mathrm{H}$ and ${ }_{3}^{6} \mathrm{Li}$. The line $A / Z=3 / 2$ of the star mass spectrum can not be formed by these nuclei. However, the reaction

$$
{ }_{6}^{9} C+e \rightarrow{ }_{5}^{9} B+\widetilde{\nu},
$$

is not energetically allowed and therefore it is possible to believe that the stars of the above line mass spectrum may consist of carbon-9.

It is interesting, is it possible to study this effect under laboratory conditions? One can use gaseous tritium for it and can measure the rate of its decay at two states. First the rate of tritium decay at atomic (molecular) state. Second, the same rate under the effect of ionizing electric discharge. Essentially, one must aim to use the dense gas and the power discharge in order to ionize more atoms of tritium. Taking into account the preceding consideration, such a test does not look as a hopeless project.

The mechanism of neutronization acting in the nondegenerate dense plasma and described above in this chapter 


\begin{tabular}{|l|l:c|}
\hline $\begin{array}{l}\text { Atomic } \\
\text { substance }\end{array}$ & $\begin{array}{c}\text { Low T } \\
\text { body }\end{array}$ & High T \\
\hline Plasma & Non-relativistic & Non-relarivistic \\
$P_{F}<<m_{e} c$ & degenerate & Non-degenerate \\
\hline $\begin{array}{l}\text { Plasma } \\
P_{F} \approx m_{e} c\end{array}$ & relativistic & $\begin{array}{l}\text { relativistic } \\
\text { degenerate }\end{array}$ \\
\hline $\begin{array}{c}\text { neutron } \\
\text { matter }\end{array}$ & relativistic & $\begin{array}{l}\text { relativistic } \\
P_{F} \approx m_{n} c\end{array}$ \\
degenerate & non-degenerate \\
\hline \hline
\end{tabular}

Figure 1. The steady states of substance

seems quite realistic. However, the last nuclear reaction of neutronization can only be considered as hypothetical possibility and requires further more careful study.

\section{Other stars, their classification and some cosmology}

The Schwarzsprung-Rassel diagram is now a generally accepted base for star classification. It seems that a classification based on the EOS of substance may be more justified from physical point of view. It can be emphasized by possibility to determine the number of classes of celestial bodies.

The matter can totally have eight states (Fig.(1)).

The atomic substance at low temperature exists as condensed matter (solid or liquid). At high temperature it transforms into gas phase.

The electron -nuclear plasma can exist in four states. It can be relativistic or non-relativistic. The electron gas of non-relativistic plasma can be degenerate (cold) or nondegenerate (hot). The relativistic electron gas is degenerate at temperature below $T_{F}$. Very high temperature can remove the degeneration even for relativistic electrons (if, certainly, electronic gas was not ultra-relativistic originally).

In addition to that, a substance can exist as neutron matter with the nuclear density approximately.

At present, assumptions about existence of matter at different states, other than the above-named, seem unfounded. Thus, these above-named states of matter show a possibility of classification of celestial bodies in accordance with this dividing.

\subsection{The atomic substance}

\subsubsection{Small bodies}

Small celestial bodies - asteroids and satellites of planets are usually considered as bodies consisting from atomic matter.

\subsubsection{Giants}

The transformation of atomic matter into plasma can be induced by action of high pressure, high temperature or both these factors. If these factors inside a body are not high enough, atomic substance can transform into gas state. The characteristic property of this celestial body is absence of electric polarization inside it. If temperature of a body is below ionization temperature of atomic substance but higher than its evaporation temperature, the equilibrium equation comes to

$$
-\frac{d P}{d r}=\frac{G \gamma}{r^{2}} M_{r} \approx \frac{P}{R} \approx \frac{\gamma}{m_{p}} \frac{k T}{R} .
$$

Thus, the radius of the body

$$
R \approx \frac{G M m_{p}}{k T}
$$

If its mass $M \approx 10^{33} \mathrm{~g}$ and temperature at its center $T \approx$ $10^{5} \mathrm{~K}$, its radius is $R \approx 10^{2} R_{\odot}$. These proporties are characteristic for giants, where pressure at center is about $P \approx 10^{10} \mathrm{din} / \mathrm{cm}^{2}$ and it is not enough for substance ionization.

\subsection{Plasmas}

\subsubsection{The non-relativistic non-degenerate plasma. Stars.}

Characteristic properties of hot stars consisting of nonrelativistic non-degenerate plasma was considered above in detail. Its EOS is ideal gas equation.

\subsection{Non-relativistic degenerate plasma. Plan- ets.}

At cores of large planets, pressures are large enough to transform their substance into plasma. As temperatures are not very high here, it can be supposed, that this plasma can degenerate:

$$
T<<T_{F}
$$

The pressure which is induced by gravitation must be balanced by pressure of non-relativistic degenerate electron gas

$$
\frac{G \mathbb{M}^{2}}{6 \mathbb{R} \mathbb{V}} \approx \frac{\left(3 \pi^{2}\right)^{2 / 3}}{5} \frac{\hbar^{2}}{m_{e}}\left(\frac{\gamma}{m_{p} A / Z}\right)^{5 / 3}
$$

It opens a way to estimate the mass of this body:

$$
\mathbb{M} \approx \mathbb{M}_{C h}\left(\frac{\hbar}{m c}\right)^{3 / 2}\left(\frac{\gamma}{m_{p}}\right)^{1 / 2} \frac{6^{3 / 2} 9 \pi}{4(A / Z)^{5 / 2}}
$$

At density about $\gamma \approx 1 \mathrm{~g} / \mathrm{cm}^{3}$, which is characteristic for large planets, we obtain their masses

$$
\mathbb{M} \approx 10^{-3} \frac{\mathbb{M}_{C h}}{(A / Z)^{5 / 2}} \approx \frac{4 \cdot 10^{30}}{(A / Z)^{5 / 2}} g
$$

Thus, if we suppose that large planets consist of hydrogen $(\mathrm{A} / \mathrm{Z}=1)$, their masses must not be over $4 \cdot 10^{30} \mathrm{~g}$. It is in agreement with the Jupiter's mass, the biggest planet of the Sun system. 


\subsubsection{The cold relativistic substance}

The ratio between the main parameters of hot stars was calculated with the using of the virial theorem (Sec.5, Part I). It allowed us to obtain the potential energy of a star, consisting of a non-relativistic non-degenerate plasma at equilibrium conditions. This energy was obtained with taking into account the gravitational and electrical energy contribution. Because this result does not depend on temperature and plasma density, we can assume that the obtained expression of the potential energy of a star is applicable to the description of stars, consisting of a degenerate plasma. At least obtained expression Eq.(I-70) should be correct in this case by order of magnitude. Thus, in view of Eq.(I-57) and Eq.(I-94), the potential energy of the cold stars on the order of magnitude:

$$
\mathcal{E}^{\text {potential }} \approx-\frac{G \mathbb{M}_{C h}^{2}}{\mathbb{R}_{0}}
$$

\section{A relativistic degenerate electron-nuclear plasma.} Dwarfs

It is characteristic for a degenerate relativistic plasma that the electron subsystem is relativistic, while the nuclear subsystem can be quite a non-relativistic, and the main contribution to the kinetic energy os star gives its relativistic electron gas. Its energy was obtained above (Eq.(5)).

The application of the virial theorem gives the equation describing the existence of equilibrium in a star consisting of cold relativistic plasma:

$$
\left[\xi\left(2 \xi^{2}+1\right) \sqrt{\xi^{2}+1}-\operatorname{Arcsinh}(\xi)-\frac{8}{3} \xi^{3}\right] \approx \xi,
$$

This equation has a solution

$$
\xi \approx 1
$$

The star, consisting of a relativistic non-degenerate plasma, in accordance with Eq.((2)), must have an electronic density

$$
n_{e} \approx 5 \cdot 10^{29} \mathrm{~cm}^{-3}
$$

while the radius of the star will

$$
R \approx 10^{-2} R_{\text {odot }}
$$

Easy to see that the density of matter and the radius are characteristic of cosmic bodies, called dwarfs.

The neutron matter. Pulsars.

Dwarfs may be considered as stars where a process of neutronization is just beginning. At a nuclear density, plasma turns into neutron matter. ${ }^{2}$

At taking into account the above assumptions, the stars composed of neutron matter, must also have the same equilibrium condition Eq.(44). As the density of neutron matter:

$$
n_{n}=\frac{p_{F}^{3}}{3 \pi^{2} \hbar^{3}}=\frac{\xi^{3}}{3 \pi^{2}}\left(\frac{m_{n} c}{\hbar}\right)^{3}
$$

(where $m_{n}$ - the neutron mass), then the condition ((44)) allows to determine the equilibrium density of matter within a neutron star

$$
n_{n}=n_{n} \approx 4 \cdot 10^{39}
$$

\footnotetext{
${ }^{2}$ At nuclear density neutrons and protons are indistinguishable inside pulsars as inside a huge nucleus. It permits to suppose a possibility of gravity induced electric polarization in this matter.
}

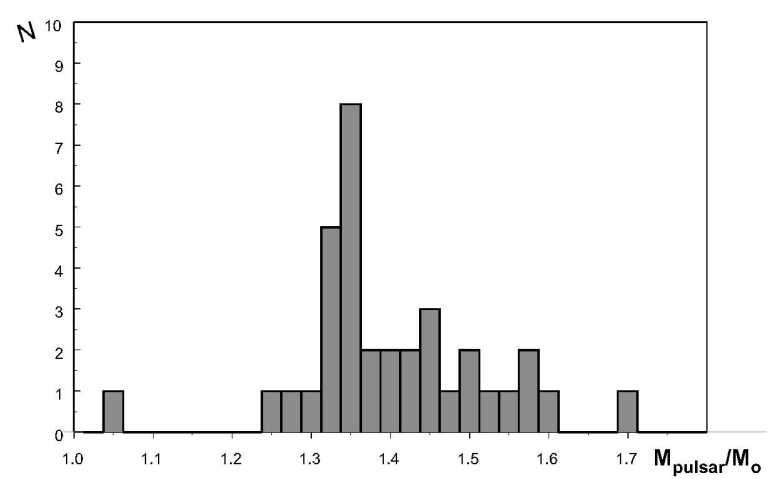

Figure 2. The mass distribution of pulsars from binary systems [5].On abscissa the logarithm of pulsar mass in solar mass is shown.

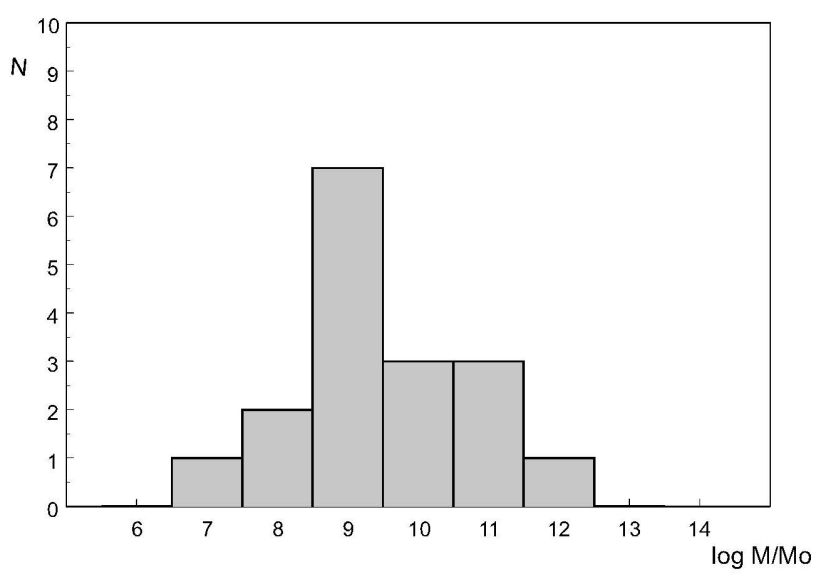

Figure 3. The mass distribution of galaxies. [6]. On the abscissa, the logarithm of the galaxy mass over the Sun mass is shown.

particles in $\mathrm{cm}^{3}$. The substitution of the values of the neutron density in the equilibrium condition Eq.(I-59) shows that, in accordance with our assessment of all the neutron stars in the steady state should have a mass of order of magnitude equal to the mass of the Sun. The measured mass distribution of pulsar composing binary stars [5] is shown on Fig.2. It can be considered as a confirmation of the last conclusion.

\subsubsection{The hot relativistic plasma.Quasars}

Plasma is hot if its temperature is higher than degeneration temperature of its electron gas. The ratio of plasma temperature in the core of a star to the temperature of degradation of its electron gas for case of non-relativistic hot star plasma is (Eq.(I-27))

$$
\frac{\mathbb{T}_{\star}}{T_{F}\left(n_{\star}\right)} \approx 40
$$

it can be supposed that the same ratio must be characteristic for the case of a relativistic hot star. At this temperature, the radiation pressure plays a main role and accordingly the equation of the pressure balance takes the form:

$$
\frac{G M^{2}}{6 R V} \approx \frac{\pi^{2}}{45} \frac{\left(k \mathbb{T}_{\star}\right)^{4}}{(\hbar c)^{3}} \approx\left(\frac{\mathbb{T}_{\star}}{T_{F}}\right)^{3} k T n
$$

This makes it possible to estimate the mass of a hot relativistic star

$$
M \approx\left(\frac{T_{\star}}{T_{F}}\right)^{6}\left(\frac{\hbar c}{G m_{p}^{2}}\right)^{3 / 2} m_{p} \approx 10^{9} M_{\odot}
$$




\begin{tabular}{|l|l|c|}
\hline & Low T & High T \\
\hline $\begin{array}{c}\text { Atomic } \\
\text { substance }\end{array}$ & asteroid & gaint \\
\hline Plasma & planet & star \\
$p_{F} \ll m_{e} c$ & & \\
\hline Plasma & & quasar \\
$p_{F} \approx m_{e} c$ & dwarf & \\
\hline $\begin{array}{l}\text { neutron } \\
\text { matter } \\
p_{F} \approx m_{n} c\end{array}$ & pulsar & \\
\hline
\end{tabular}

Figure 4. The categorization of celestial bodies

According to the existing knowledge, among compact celestial objects only quasars have masses of this level. Apparently it is an agreed-upon opinion that quasars represent some relatively short stage of evolution of galaxies. If we adhere to this hypothesis, the lack of information about quasar mass distribution can be replaced by the distribution of masses of galaxies [6](Fig.3). It can be seen, that this distribution is in a qualitative agreement with supposition that quasars are composed from the relativistic hot plasma.

Certainly the used estimation Eq.(49) is very arbitrary. It is possible to expect the existing of quasars which can have substantially lesser masses.

As the steady-state particle density of relativistic matter $n_{r}$ is known (Eq.(45)), one can estimate the quasar radius:

$$
R_{q u} \approx \sqrt[3]{\frac{M_{q u}}{n_{r} m_{p}}} \approx 10^{12} \mathrm{~cm}
$$

It is in agreement with the astronomer measuring data obtained from periods of the their luminosity changing.

\subsubsection{About the cosmic object categorizations}

Thus, it seems possible under some assumptions to find characteristic parameters of different classes of stars, if to proceed from EOS of atomic, plasma and neutron substances. The EOS types can be compared with classes of celestial bodies. As any other EOS are unknown, it gives a reason to think that all classes of celestial bodies are discovered (Fig.4).

\subsection{Several words about star evolution}

There are not formulas in this section, which could serve as a handhold for suggestions. The formulas of the previous sections can not help too in understanding that, as the evolution of the stars can proceed and as their transitions them from the one class to another are realized, since these formulas were received for the description of the star steady-state. There the comparison of the schemes to the substance categorization
(Fig.(1)) and the categorization of star classes (Fig.(4)) can serve as the base of next suggestions.

At analyzing of these schemes, one can suppose that the evolution of stellar objects is going at the reduction of their temperature. In light of it, it is possible to suppose the existing of one more body, from which the development began. Really, neutron matter at nuclear density (Eq.(48)) is not ultra-relativistic. At this density, corresponding to $p_{F} \approx m c$, its energy and pressure can depend on the temperature if this temperature it is high enough. ${ }^{3}$ It seems, there is no thermodynamical prohibition to imagine this matter so hot when the neutron gas is non-degenerate. An estimation shows that it can be possible if mass of this body is near to $10^{50} \mathrm{~g}$ or even to $10^{55} \mathrm{~g}$. As it is accepted to think that full mass of the Universe is about $10^{53} \mathrm{~g}$, it can be assumed that on an early stage of development of Universe, there was some celestial body with the mass about $10^{53} \mathrm{~g}$ composed by the neutron matter at the approximately nuclear density with at the temperature above $10^{12} \mathrm{~K}$. After some time, with temperature decreased it has lost its stability and decayed into quasars with mass up to $10^{12} M_{C h}$, consisting of the non-degenerate relativistic plasma at $T>10^{10} \mathrm{~K}$. After next cooling at loosing of stability they was decaying on galaxies of hot stars with mass about $M \approx M_{C h}$ and core temperature about $T \approx 10^{7} \mathrm{~K}$, composed by non-relativistic hot plasma. A next cooling must leads hot stars to decaying on dwarfs, pulsars, planets or may be on small bodies. The substances of these bodies (in their cores) consists of degenerate plasma (degenerate electron subsystem and cold nuclear subsystem) or cold neutron matter, it makes them stable in expanding and cooling Universe. ${ }^{4}$ It is important to emphasize, that the gravity induced electric polarization excludes the possibility of the gravitational collapse as the last stage of the star evolutions.

\subsection{About $\ll$ black holes $\gg$}

It seems that the idea about the «black holes》 existence is organic related to the suggestion about an inevitable collapse of large cosmic bodies on the last stage of their evolutions. However, the models of collapsing masses were appearing as a consequence of the rejection from attention of the gravity induced electric polarization of the intra-stellar plasma, which was considered in previous chapters. If to take into account this mechanism, the possibility of collapse must be excludes. It allows newly to take a look on the $\ll$ black hole $\gg$ problem.

In accordance with the standard approach, the Schwarzshield radius of «black hole» with $\mathrm{M}$ is

$$
r_{b h}=\frac{2 G M}{c^{2}}
$$

and accordingly the average density of $\ll$ black holes $\gg$ :

$$
\gamma_{b h}=\frac{3 c^{6}}{32 \pi M^{2} G^{3}} .
$$

The estimations which was made in previous chapters are showing that all large inwardly-galactic objects of all classes - a stars, dwarves, pulsars, giants - possess the mass of the

\footnotetext{
${ }^{3}$ The ultra-relativistic matter with $p_{F} \gg m c$ is possessed by limiting pressure which is not depending on temperature.

${ }^{4}$ The temperature of plasma inside these bodies can be really quite high as electron gas into dwarfs, for example, will be degenerate even at temperature $T \approx 10^{9} \mathrm{~K}$.
} 
order $M_{C h}$ (or $10 M_{C h}$ ). As the density of these objects are small relatively to the limit Eq.(54). As result, a searching of «black hole» inside stellar objects of our Galaxy seems as hopeless.

On the other hand, stellar objects, consisting of hot relativistic plasma - a quasars, in accordance with their mass and density, may stay «black holes》. The process of collapse is not needed for their creation. As the quasar mass $M_{q u} \gg M_{C h}$, all other stellar objects must organize their moving around it and one can suppose that a $\ll$ black hole» can exist at the center of our Galaxy.

\section{The conclusion}

Evidently, the main conclusion from the above consideration consists in statement of the fact that now there are quite enough measuring data to place the theoretical astrophysics on a reliable foundation. All above measuring data are known for a relatively long time. The traditional system of view based on the Euler equation in the form Eq.(I-1) could not give a possibility to explain and even to consider. with due proper attention, to these data. Taking into account the gravity induced electric polarization of plasma and a change starting postulate gives a possibility to obtain results for explanation of measuring data considered above.

Basically these results are the following.

Using the standard method of plasma description leads to the conclusion that at conditions characteristic for the central stellar region, the plasma has the minimum energy at constant density $n_{\star}$ (Eq.(I-22)) and at the constant temperature $\mathbb{T}_{\star}$ (Eq.(I-78).

This plasma forms the core of a star, where the pressure is constant and gravity action is balanced by the force of the gravity induced by the electric polarization. The virial theorem gives a possibility to calculate the stellar core mass $\mathbb{M}_{*}$ (Eq.(I-82)) and its radius $\mathbb{R}_{\star}$ Eq.(I-84). At that the stellar core volume is approximately equal to $1 / 1000$ part of full volume of a star.

The remaining mass of a star located over the core has a density approximately thousand times smaller and it is convenient to name it a star atmosphere. At using thermodynamical arguments, it is possible to obtain the radial dependence of plasma density inside the atmosphere $n_{a} \approx r^{-6}$ (Eq.(I54)) and the radial dependence of its temperature $\mathbb{T}_{a} \approx r^{-4}$ (Eq.(I-55)).

It gives a possibility to conclude that the mass of the stellar atmosphere $\mathbb{M}_{a}$ (Eq.(I-56)) is almost exactly equal to the stellar core mass.Thus, the full stellar mass can be calculated. It depends on the ratio of the mass and the charge of nuclei composing the plasma. This claim is in a good agreement with the measuring data of the mass distribution of both - binary stars and close binary stars (Fig.(I-1)-Fig.(I-2)) ${ }^{5}$. At that it is important that the upper limit of masses of both binary stars and close binary stars - is in accordance with the calculated value of the mass of the hydrogen star (Eq.(I-83)).

\footnotetext{
${ }^{5}$ The measurement of parameters of these stars has a satisfactory accuracy only.
}

The obtained formula explains the origin of sharp peaks of stellar mass distribution - they evidence that the substance of these stars have a certain value of the ratio $A / Z$. In particular the solar plasma according to (Eq.(I-83)) consists of nuclei with $A / Z=5$.

Knowing temperature and substance density on the core and knowing their radial dependencies, it is possible to estimate the surface temperature $\mathbb{T}_{0}$ Eq.(I-95) and the radius of a star $\mathbb{R}_{0}$ Eq.(I-94). It turns out that these measured parameters must be related to the star mass with the ratio $\mathbb{T}_{0} \mathbb{R}_{0} \sim \mathbb{M}^{5 / 4}$ Eq.(I-103). It is in a good agreement with measuring data (Fig.(I-3)).

Using another thermodynamical relation - the Poisson's adiabat - gives a way to determine the relation between radii of stars and their masses $\mathbb{R}_{0}^{3} \sim \mathbb{M}^{2}$ (Eq.(II-16)), and between their surface temperatures and masses $\mathbb{T}_{0} \sim \mathbb{M}^{5 / 7}$ (Eq.(II-20)). It gives the quantitative explanation of the massluminosity dependence (Fig.(II-3)).

According to another familiar Blackett's dependence, the giromagnetic ratios of celestial bodies are approximately equal to $\sqrt{G} / c$. It has a simple explanation too. When there is the gravity induced electric polarization of a substance of a celestial body, its rotation must induce a magnetic field (Fig.(II-5)). It is important that all (composed by eN-plasma) celestial bodies - planets, stars, pulsars - obey the Blackett's dependence. It confirms a consideration that the gravity induced electric polarization must be characterizing for all kind of plasma. The calculation of magnetic fields of hot stars shows that they must be proportional to rotation velocity of stars Eq.(II-43). Magnetic fields of Ap-stars are measured, and they can be compared with periods of changing of luminosity of these stars. It is possible that this mechanism is characteristic for stars with rapid rotation (Fig.(II-6)), but obviously there are other unaccounted factors.

Taking into account the gravity induced electric polarization and coming from the Clairault's theory, we can describe the periastron rotation of binary stars as effect descended from non-spherical forms of star cores. It gives the quantitative explanation of this effect, which is in a good agreement with measuring data (Fig.(II-7)).

The solar oscillations can be considered as elastic vibrations of the solar core. It permits to obtain two basic frequencies of this oscillation: the basic frequency of sound radial oscillation of the core and the frequency of splitting depending on oscillations of substance density near its equilibrium value (Fig.(II-9)).

The plasma can exists in four possible states. The nonrelativistic electron gas of plasma can be degenerate and non-degenerate. Plasma with relativistic electron gas can have a cold and a hot nuclear subsystem. Together with the atomic substance and neutron substance, it gives seven possible states. It suggests a way of a possible classification of celestial bodies. The advantage of this method of classification is in the possibility to estimate theoretically main parameters characterizing the celestial bodies of each class. And these predicted parameters are in agreement with astronomical observations. It can be supposed hypothetically that 
cosmologic transitions between these classes go in direction of their temperature being lowered. But these suppositions have no formal base at all.

Discussing formulas obtained, which describe star properties, one can note a important moment: these considerations permit to look at this problem from different points of view. On the one hand, the series of conclusions follows from existence of spectrum of star mass (Fig.(I-1)) and from known chemical composition dependence. On the another hand, the calculation of natural frequencies of the solar core gives a different approach to a problem of chemical composition determination. It is important that for the Sun, both these approaches lead to the same conclusion independently and unambiguously. It gives a confidence in reliability of obtained results.

In fact, the calculation of magnetic fields of hot stars agrees with measuring data on order of the value only. But one must not expect the best agreement in this case because calculations were made for the case of a spherically symmetric model and measuring data are obtained for stars where this symmetry is obviously violated. But it is important, that the all remaining measuring data (all known of today) confirm both - the new postulate and formulas based on it. At that, the main stellar parameters - masses, radii and temperatures - are expressed through combinations of world constants and at that they show a good accordance with observation data. It is important that quite a satisfactory quantitative agreement of obtained results and measuring data can be achieved by simple and clear physical methods without use of any fitting parameter. It gives a special charm and attractiveness to star physics.

\section{REFERENCES}

[1] Landau L.D. and Lifshits E.M.: Statistical Physics, 1, 3rd edition, Oxford:Pergamon, (1980)

[2] Leung Y.C.: Physics of Dense Matter In Science Press/World Scientific, Beijing and Singapore, (1984)

[3] Landau L.D. and Lifshits E.M.: The Classical Theory of Fields. 1, Pergamon Press, N.Y. (1971)

[4] BlochJ., Mintz H. : Kinetics and mechanisms of metal hydrides formation - a review. J. of Alloys and Compound. v.253-254, pp.529-541 (1997)

[5] Thorsett S.E. and Chakrabarty D.: E-preprint: astro-ph/9803260, (1998)

[6] Allen C.W. - Astrophysical quantities, 1955,University of London, The Athlone Press.

[7] Khaliullin K.F.: Dissertation, Sternberg Astronomical Institute, Moscow, (Russian)(2004) (see Table in Appendix on http://astro07.narod.ru) 


\section{Appendix}

The Table of main parameters of close binary stars (cited on Kh.F.Khaliullin's dissertation, Sternberg Astronomical Institute. In Russian)[7]

\begin{tabular}{|c|c|}
\hline 峞 & 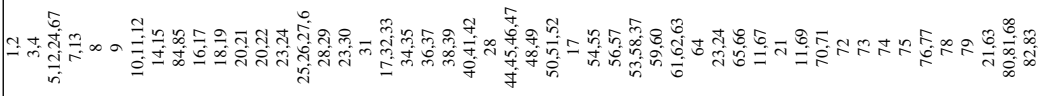 \\
\hline 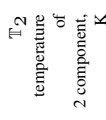 & 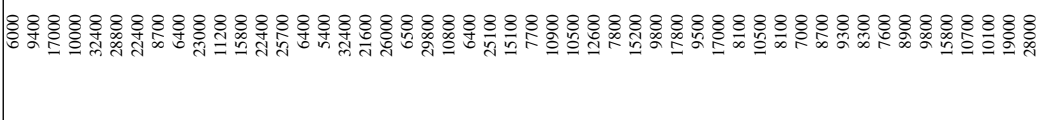 \\
\hline 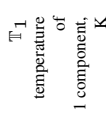 & 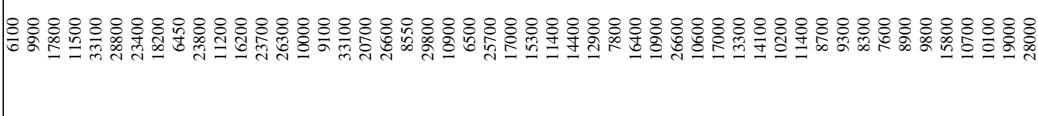 \\
\hline 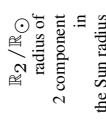 & 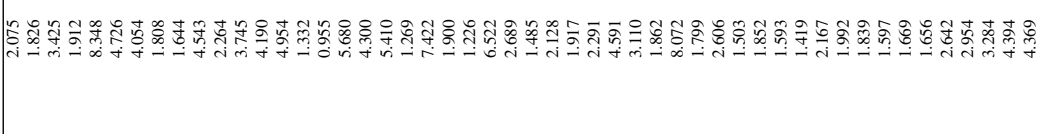 \\
\hline 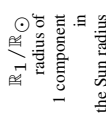 & 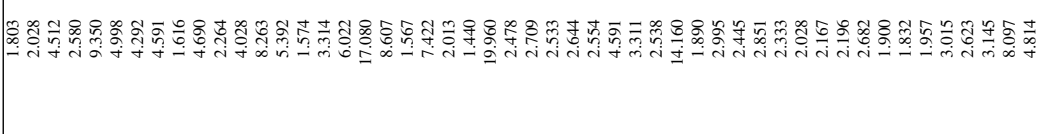 \\
\hline 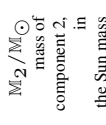 & 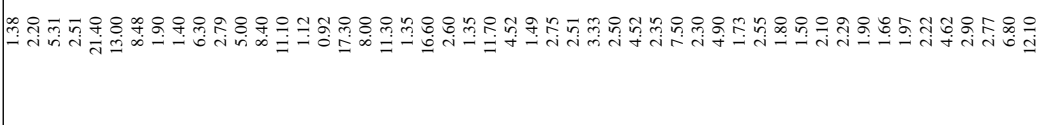 \\
\hline 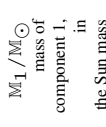 & 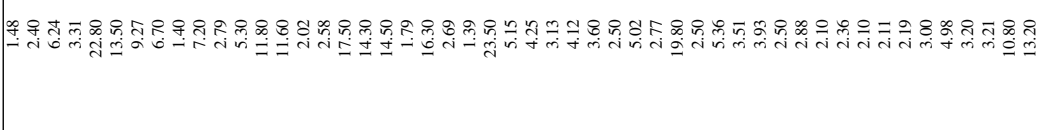 \\
\hline 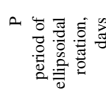 & 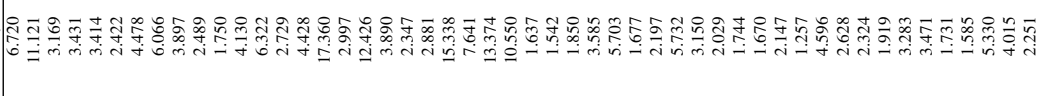 \\
\hline 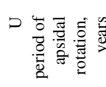 & 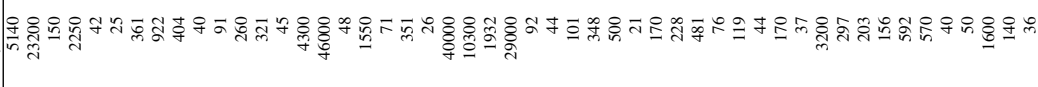 \\
\hline 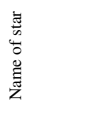 & 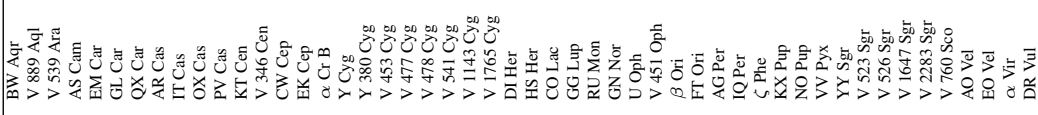 \\
\hline z & - \\
\hline
\end{tabular}




\section{REFERENCES}

[1] Khaliulilin Kh.F. and Kozyreva V.S.

Apsidal motion in the eclipsing binary system of $B W$ Aqr

Astrophys. and Space Sci., 120 (1986) 9-16.

[2] Imbert $\mathbf{M}$.

Photoelectric radial velosities of eclipsing binaries. IV. Orbital elements of BW Aqr,

Astron.Astrophys.Suppl., 69 (1987) 397-401.

[3] Khaliulilin Kh.F. and Khaliulilina A.I.

Fotometricheskoe issledovanie zatmenno-dvoinoi sistemy s relativistskim vrasheniem orbity V889 Aql,

Astronom.zh.,66(1989)76-83 (in Russian).

[4] Khaliulilin Kh.F. and Khaliulilina A.I.

$K$ probleme vrashenia linii apsid $v$ zatmennoi sisteme V889 Aql,

Astron.cirk., N1486 (1987) 5-7 (in Russian).

[5] Clausen J.V.

V 539 Arae: first accurate dimensions of eclipsing binaries,

Astron.Astrophys., 308 (1996) 151-169.

[6] Lavrov M.I. and Lavrova N.V.

Revisia elementov fotometricheskoi orbity EK Cep,

Astron.cirk. 971 (1977) 3-4 (in Russian).

[7] Khaliulilin Kh.F. and Kozyreva V.S. Apsidal motion in the eclipsing binary AS Cam,

Astrophys. and Space Sci., 120 (1994) 115-122.

[8] Andersen J. and Clausen J.V., Absolute dimensions of eclipsing binaries.XV. EM Cainae,

Astron.Astrophys. 213 (1989) 183-194.

[9] Gemenez A. and Clausen J.V.,

Four-color photometry of eclipsing binaries. XXIIA. Photometric elements and apsidal motion of GL Cainae,

Astron.Astrophys. 161 (1986) 275-286.

[10] Andersen J., Clausen J.V., Nordstrom B. and Reipurth B.,

Absolute dimensions of eclipsing binaries.I. The earlytype detached system QX Cainae,

Astron.Astrophys. 121 (1983) 271-280.

[11] Gemenez A., Clausen J.V. and Jensen K.S.

Four-color photometry of eclipsing binaries. XXIV. Aspidal motion of QX Cainae, $\xi$ Phoenicis and NO Puppis,

Astron.Astrophys. 159 (1986) 157-165.

[12] De Greve J.P.

Evolutionary models for detached close binaries: the system Arae and QX Cainae,

Astron.Astrophys. 213 (1989) 195-203.

[13] Malony F.P., Guinan E.F. and Mukherjec J.

Eclipsing binary star as test of gravity theories

Astron.J. 102 (1991) 256-261.

[14] Mossakovskaya L.V.

New photometric elements of AR Cas, an eclipsing binary system with apsidal motion

Astron. and Astroph. Trans. 2 (1992) 163-167.

[15] Haffer C.M. and Collins G.M.

Computation of elements of eclipsing binary stars by high-speed computing machines

Astroph.J.Suppl., 7 (1962) 351-410.

[16] Crinklaw G. and Etzel P.

A photometric analisis of the eclipsing binary OX Cassiopeiae

Astron.J. 98 (1989) 1418-1426.

[17] Claret A. and Gimenez A.

The aspidal motion test of the internal stellar structure: comparision between theory and observations

Astron.Astroph. 277 (1993) 487-502.

[18] Wolf M.

Aspidal motion in the eclipsing binary PV Cassiopeiae Monthly Not.Roy.Soc. 286 (1995) 875-878.

[19] Popper D.M.

Rediscussion of eclipsing binaries.XVII.The detached early A type binaries PV Cassiopeae and WX Cephei

Astron.J. 93 (1987) 672-677.

[20] Lavrov M.I. and Lavrova N.V.

Revisia fotometrichestih elementov $u$ zatmennyh dvoinyh sistem s ekscentricheskimi orbitami.2.KT Cen Trudy Kaz.Gor.AO 49 (1985) 18-24 (in Russian).

[21] Soderhjelm S.

Observations of six southern eclipsing binaries for apsidal motion

Astron.Astroph.Suppl.Ser 22 (1975) 263-283.

[22] Gemenez A., Clausen J.V. and Anderson J.

Four-color photometry of eclipsing binaries. XXIA. Photometric analysis and aspidal motion study of V346 Centauri,

Astron.Astrophys. 160 (1986) 310-320.

[23] Gemenez A., Chun-Hwey Kim and Il-Seong Nha Aspidal motion in the early-type eclipsing binaries $C W$ Cephei, Y Cyg and AG Per

Montly .Not.Roy.Astron.Soc. 224 (1987) 543-555. 
[24] Bocula R.A.

Peresmotr elementov fotometricheskoi orbity zatmennyh sistem CW Cep, V 539 Ara, AG Per, AR Aur, RS Cha, ZZ Boo.

Peremennye zvezdy21 (1983) 851-859 (in Russian).

[25] Khaliulilin Kh.F.

Relativistskoe vrashenie orbity zatmennoi dvoinoi sistemy EK Cep

Astron.zh.60 (1983) 72-82 (in Russian).

[26] Tomkin J.

Secondaries of eclipsing binary. V. EK Cephei

Astroph.J. 271 (1983) 717-724.

[27] Claret A., Gemenez A. and Martin E.L.

A test case of stellar evolution the eclipsing binary EK Cephei

Astron.Astroph. 302 (1995) 741-744.

[28] Volkov I.M.

The discovery of apsidal motion in the eclipsing binary system $\alpha \mathrm{Cr} B$

Inf.Bull.Var.Stars N3876,(1993) 1-2.

[29] Quiroga R.J., van L.P.R.

Angular momenta in binary systems

Astroph.Space Sci. 146 (1988) 99-137.

[30] Hill G. and Holmgern D.E.

Studies of early-type varieble stars

Asrton.Astroph.297 (1995) 127-134.

[31] Hill G. and Batten A.H.

Studies of early-type varieble stars.III. The orbit and physical dimensions for $V 380$ Cygni

Asrton.Astroph.141 (1984) 39-48.

[32] Zakirov M.M.

Ob apsidalnom dvizhenii v dvoinoi sisteme V 453 Cyg

Astron.cirk.N1537,21 (in Russian).

[33] Karetnikov V.G.

Spectral investigation of eclipsing binary stars at the stage of mass exchange

Publ.Astron.Inst.Czech.70 (1987) 273-276.

[34] Mossakovskaya L.V. and Khaliulilin Kh.F.

Prichina anomalnogo apsidalnogo dvizhenia $v$ sisteme V 477 Cyg

Astron.cirk.N1536, 23-24 (in Russian).

[35] Gemenez A. and Quintana J.M.

Apsidal motion and revised photometry elements of the eccentric eclipsing binary V 477 Cyg

Astron.Astrophys. 260 (1992) 227-236.

[36] Mossakovskaya L.V. and Khaliulilin Kh.F.

Vrashenie linii apsid v sisteme V 478 Cyg

Pisma v Astron.zh.22 (1996) 149-152.
[37] Popper D.M. and Hill G.

Rediscussion of eclipsing binaries.XVII.Spectroscopic orbit of $O B$ system with a cross-correlation procedure

Astron.J. 101 (1991) 600-615.

[38] Khaliulilin Kh.F.

The unique eclipsing binary system V 541 Cygni with relativistic apsidal motion

Astrophys.J. 229 (1985) 668-673.

[39] Lines R.D.,Lines H., Guinan E.F. and Carroll

Time of minimum determination of eclipsing binary $V$ 541 Cygni

Inf.Bull.Var.Stars N3286,1-3.

[40] Khaliulilin Kh.F.

Vrashenie linii apsid v zatmennoi sisteme V 1143 Cyg Asrton. cirk.N1262,1-3 (in Russian).

[41] Andersen J., Garcia J.M., Gimenes A. and Nordstom B. Absolute dimension of eclipsing binaries.X. V1143 Cyg Astron.Astrophys. 174 (1987) 107-115.

[42] Burns J.F., Guinan E.F. and Marshall J.J.

New apsidal motion determination of eccentric eclipsing binary V 1143 Cyg

Inf.Bull.Var.Stars N4363,1-4.

[43] Hill G. and Fisher W.A.

Studies of early-type varieble stars.II. The orbit and masses ofHR 7551

Astron.Astrophys. 139 (1985) 123-131.

[44] Martynov D.Ya. and Khaliulilin Kh.F.

On the relativistic motion of periastron in the eclipsing binary system DI Her

Astrophys.and Space Sci. 71 (1980) 147-170.

[45] Popper D.M.

Rediscussion of eclipsing binaries.XVII. DI Herculis, a $B$-tipe system with an accentric orbit

Astron.J. 254 (1982) 203-213.

[46] Martynov D.Ia. Lavrov M.I.

Revizia elementov fotometricheskoi orbity $i$ skorost vrashenia linii apsid u zatmennoi dvoinoi sistemy DI $\mathrm{Her}$

Pisma v Astron.Zh. 13 (1987) 218-222 (in Russian).

[47] Khaliulilin Kh.F., Khodykin S.A. and Zakharov A.I.

On the nature of the anomalously slow apsidal motion of DI Herculis

Astrophys.J. 375 (1991) 314-320.

[48] Khaliulilina A.I. and Khaliulilin Kh.F.

Vrashenie linii apsid $v$ zatmennoi dvoinoi sisteme HS $\mathrm{Her}$

Astron.cirk.N 1552 (1992) 15-16(in Russian). 
[49] Martynov D.Ia., Voloshina I.E. and Khaliulilina A.I. Fotometricheskie elementy zatmennoi sistemy HS Her Asrton. zh. 65 (1988) 1225-1229 (in Russian).

[50] Mezzetti M., Predolin F., Giuricin G. and Mardirossian F.

Revised photometric elements of eight eclipsing binaries

Astron.Astroph.Suppl. 42 (1980) 15-22.

[51] Mossakovskaya L.V. and Khaliulilin Kh.F.

Tret'e telo $v$ zatmennoi sisteme s apsidalnym dvizheniem CO Lac?

Astron. cirk.N1495, 5-6 (in Russian).

[52] Semeniuk I.

Apsidal motion in binary systems. I. CO Lacertae, an eclipsing variable with apsidal motion

Acta Astron. 17 (1967) 223-224.

[53] Andersen J.

Accurate masses and radii of normal stars

Astron.Astroph.Rev. 3 (1991) 91-126.

[54] Khaliulilina A.I., Khaliulilin Kh.F. and Martynov D.Ya. Apsidal motion and the third body in the system $R U$ Monocerotis

Montly .Not.Roy.Astron.Soc. 216 (1985) 909-922.

[55] Martynov D.Ya. and Khaliulilina A.I.

RU Monocerotis: poslednie resultaty

Astron.zh.63 (1986) 288-297 (in Russian).

[56] Shneller H.

Uber die periodenanderrungen bei bedeckungsveranderlichen

Budd.Mitt. N53 (1962) 3-41.

[57] Kort S.J., J. de, The orbit and motion of priastron of GN Normae

Ricerche Astron. 3 (1954) 119-128.

[58] Kamper B.C.

Light-time orbit and apsidal motion of eclipsing binary U Ophiuchi

Astrophys. Space Sci. 120 (1986) 167-189.

[59] Clausen J.V., Gemenez A. and Scarfe C.

Absolute dimentions of eclipsing binaries.XI. V 451 Ophiuchi

Astron.Astroph. 167 (1986) 287-296.

[60] Khaliulilin Kh.F. and Kozyreva V.S.

Photometric light curves and physical parameters of eclipsing binary systems IT Cas, CO Cep, AI Hya with possible apsidal motions

Astrophys. and Space Sci., 155 (1989) 53-69.
[61] Monet D.G.

A discussion of apsidal motion detected in selected spectroscopic binary systems

Astrophys. J., 237 (1980) 513-528.

[62] Svechnicov M.A.

Katalog orbitalnyh elementov, mass $i$ svetimostei tesnyh dvoinyh zvezd

Irkutsk, Izd-vo Irkutsk. Univer.(In Russian).

[63] Brancewicz H.K. and Dworak T.Z.

A Catalogue of parameters for eclipsing binaries

Acta Astron., 30 (1980) 501-524.

[64] Wolf M. and Saronova L.,

Aspidal motion in the eclipsing binary FT Ori

Astron.Astroph. Suppl.114 (1995) 143-146.

[65] Drozdz M., Krzesinski J. and Paydosz G.,

Aspidal motion of IQ Persei

Inf. Bull.Var.Stars, N3494, 1-4.

[66] Lacy C.H.S. and Fruch M.L.

Absolute dimentions and masses of eclipsing binaries. V. IQ Persei

Astroph.J.295 (1985) 569-579.

[67] Andersen J.

Spectroscopic observations of eclipsing binaries. V. Accurate mass determination for the B-type systems V 539 Arae and $\xi$ Phaenicis

Astron.Astroph.118 (1983) 255-261.

[68] Odell A.P.

The structure of Alpha Virginis.II. The apsidal constant Astroph.J.192 (1974) 417-424.

[69] Gronbech B.

Four-color photometry of eclipsing binaries. V. photometric elements of NO Puppis

Astron.Astroph.50 (1980) 79-84.

[70] Harmanec P.

Stellar masses and radii based on motion binary data Bull.Astron.Inst.Czech.39 (1988) 329-345.

[71] Andersen J., Clausen L.V. and Nordstrom B.

Absolute dinemtions of eclipsing binaries. V. VV Pyxidis a detached early A-tipe system with equal components

Astron.Astroph.134 (1984) 147-157.

[72] Lacy C.H.S.

The photometric orbit and apsidal motion of YY Sagittarii

Astroph.J.105 (1993) 637-645.

[73] Lacy C.H.S.

The photometric orbit and apsidal motion of $V 523$ Sagittarii

Astroph.J.105 (1993) 630-636. 
[74] Lacy C.H.S.

The photometric orbit and apsidal motion of $V 526$ Sagittarii

Astroph.J.105 (1993) 1096-1102.

[75] Andersen J. and Gimenes A.

Absolute dinemtions of eclipsing binaries.VII. V 1647 Sagittarii

Astron.Astroph.145 (1985) 206-214.

[76] Swope H.H.

$V 2283$ Sgr, an eclipsing star with rotating apse

Ric.Astron.8 (1974) 481-490.

[77] O’Konnell D.J.K.

The photometric orbit and apsidal motion of V2283 Sagittarii

Ric.Astron.8 (1974) 491-497.

[78] Andersen J., Clausen L.V., Nordstrom B. and Popper D.M.

Absolute dinemtions of eclipsing binaries.VIII. V 760 Scorpii

Astron.Astroph.151 (1985) 329-339.

[79] Clausen L.V., Gimenez A. and Houten C.J.

Four-color photometry of eclipsing binaries.XXVII. A photometric anallysis of the (possible) Ap system AO Velorum

Astron.Astroph.302 (1995) 79-84.
[80] Popper D.M.

Stellar masses

Ann. Rev. Astron. and Astroph.18 (1980) 115-164.

[81] Dukesr R.J.

The beta Cephei nature of Spica

Astroph.J.192 (1974) 81-91.

[82] Khaliulilina A.I.

DR Vulpeculae: the quadruple system

Montly .Not.Roy.Astron.Soc. 225 (1987) 425-436.

[83] Khaliulilin Kh.F. and Khaliulilina A.I.

Fotometricheskoe issledovanie zatmennoi zvezdy DR Vul. Parametry sistemy $i$ vrashenie linii apsid,

Astron.zh., N65 (1988) 108-116 (in Russian).

[84] Khaliulilin Kh.F. and Kozyreva V.S.

Photometric light curves and physical parameters of eclipsing binary systems IT Cas, CO Cep, AI Hya with possible apsidal motions

Astrophys. and Space Sci., 155 (1989) 53-69.

[85] Holmgren D. anf Wolf M. Apsidal motion of the eclipsing binary IT Cassiopeiae

Observatory 116 (1996) 307-312. 